\title{
The hydrophobic heptad repeat in Region III of Escherichia coli transcription factor sigma 54 is essential for core RNA polymerase binding
}

\author{
Mingli Hsieh, ${ }^{1}$ Hsiu-Mei Hsu, ${ }^{1}$ Shiow-Fen Hwang, ${ }^{1}$ Feng-Chen Wen, ${ }^{2}$ \\ Ju-Shan $\mathrm{Yu}^{2}{ }^{2}$ Chun-Chiang Wen ${ }^{2}$ and Chuan $\mathrm{Li}^{1}$
}

Institute of Medicine ${ }^{1}$ and School of Medical Technology2, Chung Shan Medical and Dental College, Taichung, Taiwan, ROC
Author for correspondence: Mingli Hsieh. Tel: +88643896190 ext. 50617. Fax: + 88643892412. e-mail: mingli@mercury.csmc.edu.tw

Escherichia coli transcription factor sigma 54 contains motifs that resemble closely those used for RNA polymerase II in mammalian cells, including two hydrophobic heptad repeats, a very acidic region and a glutamine-rich region. Triple changes in hydrophobic or multiple changes in acidic residues in Region III are known to severely impair core-binding ability. To investigate whether all the changes in triple mutants are necessary for core binding, site-directed mutagenesis was performed to create single and double mutants in the leucine or isoleucine residues in the heptad repeat in Region III. Single mutants showed no discernible loss of function. Double mutants showed partial protection of the -12 promoter element of the gInAp2 promoter due to the partial loss of their ability to bind core RNA polymerase. These mutations were deleterious to the function of sigma 54, which retained only 30-40\% of wildtype mRNA levels. However, double mutants retained nearly normal ability to form open complexes. Two triple mutants created during previous work lost most, if not all, of their ability to bind core RNA polymerase, to protect the -12 promoter element of the gInAp2 promoter and to open the transcription start site. The two triple mutants produced about $20 \%$ or less than $10 \%$ of the wild-type transcripts from the gInAp2 promoter. These results demonstrate that the hydrophobic heptad repeat in Region III is essential for core RNA polymerase binding. Progressive loss of hydrophobicity of the hydrophobic heptad repeat in Region III of sigma 54 resulted in a progressive loss of corebinding ability, leading to the loss of -12 promoter element recognition and mRNA production.

Keywords : sigma 54, site-specific mutagenesis, hydrophobic heptad repeat, enhancerdependent transcription, core-binding activity

\section{INTRODUCTION}

Sigma 54 is an alternative bacterial sigma factor. In comparison to other sigma factors, sigma 54 stands alone in exhibiting no significant amino acid homology to sigma 70 (Merrick et al., 1987; Gross et al., 1992). The promoters recognized by sigma 54 polymerase are also different: instead of promoter elements covering -35 and -10 positions, the sigma 54 polymerase recognizes elements at the -24 and -12 positions. In addition, the mechanism of activation of the sigma 54 promoter is

Abbreviation: DMS, dimethyl sulfate. different in that these promoters respond to enhancer elements (Reitzer \& Magasanik, 1986; Birkmann \& Bock, 1989). The sigma 54 holoenzyme forms an unusually stable closed complex at the $g \ln A p 2$ promoter prior to its activation (Sasse-Dwight \& Gralla, 1988; Popham et al., 1989). Under nitrogen-limiting conditions, it is activated in response to the phosphorylation of NtrC (Popham et al., 1989), which binds upstream enhancer elements and contacts the bound holoenzyme by looping out the intervening DNA (Su et al., 1990, Wyman et al., 1997). This contact causes the conversion of the closed complex to the open complex state in which the start site becomes available for transcription initiation. To accomplish open complex formation, the 
activator proteins hydrolyse a nucleoside triphosphate (Weiss et al., 1992; Austin \& Dixon, 1992; Austin et al., 1994).

The Escherichia coli sigma 54 protein contains motifs reminiscent of those involved in eukaryotic enhancerdependent transcription (Sasse-Dwight \& Gralla, 1990). These include two hydrophobic heptad repeats, a very acidic region and a glutamine-rich region (Sasse-Dwight \& Gralla, 1990 and see Fig. 1). The potential roles of these regions have been investigated by assaying mutant forms of sigma 54. Previous studies of a variety of pointmutated sigma 54 proteins acting at the $g \ln A p 2$ promoter have suggested roles for the acidic residues, glutamine residues, leucine-rich motif, hydrophobicacidic region and C-terminal domain (Sasse-Dwight \& Gralla, 1990; Hsieh \& Gralla, 1994; Hsieh et al., 1994; Tintut et al., 1994; Tintut \& Gralla, 1995; Wong et al., 1994; Guo \& Gralla, 1997; Syed \& Gralla, 1998). Recognition of the -24 region appears to be dominant in terms of DNA-binding affinity (Sasse-Dwight \& Gralla, 1990; Wong et al., 1994; Cannon et al., 1995a) and the specific DNA-binding domain was localized to the C-terminus of sigma 54 (Sasse-Dwight \& Gralla, 1990; Guo \& Gralla, 1997). However, because there are two promoter elements recognized and covered by sigma 54 (Cannon et al., 1995a), DNA-binding by sigma 54 appears to be complex and the interaction between sigma 54 and DNA is also modulated by core RNA polymerase (Morris et al., 1994; Cannon et al., 1993, 1995b). It is known that mutations in various regions of sigma 54 reduce DNA-binding affinity and lead to a loss of protection of the -12 promoter region, although they do not abolish DNA binding at the -24 promoter element (Hsieh \& Gralla, 1994; Sasse-Dwight \& Gralla, 1990; Wong et al., 1994). At least five discrete sequences associated with DNA binding have been identified (Taylor et al., 1996; Guo \& Gralla, 1997). It has been noted that mutations far outside the C-terminal DNAbinding domain influence DNA binding, suggesting that intramolecular communication between domains occurs (Sasse-Dwight \& Gralla, 1990; Merrick \& Chambers, 1992; Wong et al., 1994; Cannon et al., 1995a, 1997). It was suggested that sigma 54 function is concerted by core RNA polymerase, which directs intramolecular communication within it (Sasse-Dwight \& Gralla, 1990). However, little is known about intramolecular interactions within sigma 54 or how such interactions might contribute to activity.

Comparison of the hydrophobic heptad repeat in Region III of E. coli sigma 54 (amino acids 158-179) with other sigma 54 family members shows that this region is highly conserved among E. coli., Klebsiella pneumoniae and Salmonella typhimurium. It was suggested earlier from deletion analysis in Region I and Region III that the two heptad repeat motifs act together to form a functional domain responsible for -12 promoter element interaction and positioning the activation domain (Sasse-Dwight \& Gralla, 1990). Recent studies indicated that the multiple motifs may constitute the domain required for interaction with the -12 promoter region, including the hydrophobic heptad repeat of Region I, the hydrophobic-acidic motif of Region III, the region near residue 383 and the region near residue 340 (SasseDwight \& Gralla, 1990; Merrick \& Chambers, 1992; Wong et al., 1994; Hsieh \& Gralla, 1994; Guo \& Gralla, 1997). Observations on a series of substitution mutants suggested that the alpha-helical structure in Region I is required for -12 promoter element recognition and transcription activation (Hsieh \& Gralla, 1994). One single point mutation changing leucine at amino acid 179 to serine was created and showed no discernible defect in sigma 54 functional assays (M. Hsieh \& J. D. Gralla, unpublished; Tintut et al., 1994). Mutants with triple hydrophobic changes (LHS-3, RHS-3) or a single leucine deletion (del L179) were defective in the ability to bind core RNA polymerase (Tintut et al., 1994). It was suggested that disruption of either multiple acidic or hydrophobic residues within the hydrophobic-acidic motif of Region III was accompanied by a severe loss of ability to bind core RNA polymerase (Tintut et al., 1994). In the current work, we considered whether all the changes in each of the triple mutants are necessary for core-binding ability.

It was speculated that because of the very low frequency of hydrophobic residues in the sigma 70 core-binding region, it is likely that these residues are related to a sigma-54-specific function (Tintut \& Gralla, 1995). We aimed to determine whether sigma-54-specific functions are indeed associated with the hydrophobic residues in Region III. We performed site-directed mutagenesis to create specific single and double mutants in the leucines or isoleucines of the hydrophobic residues in the heptad repeat.

\section{METHODS}

Site-directed mutagenesis. Site-specific mutations were created at leucine or isolucine residues of the heptad repeat in Region III of the rpoN (sigma 54) gene by use of the Amersham in vitro mutagenesis kit. Procedures were as described previously (Hsieh \& Gralla, 1994). Briefly, the sigma-54encoding plasmid pTH7 was digested with EcoRI/SalI and the fragments were isolated and cloned into the M13mp18 vector to form single-stranded DNA. The mutant oligonucleotides were annealed to the single-stranded DNA template and mutagenesis procedures were then conducted according to the manufacturer's protocols. The EcoRI-SalI fragment from the replicative form of $\mathrm{M} 13$, containing the desired mutation, was then isolated and cloned back into the pTH7 plasmid. The double mutants were created by annealing the single mutant oligonucleotides to the single-stranded M13 vector which contained single point mutations. The host strain, YMC109tk (thi endA hsr lacU169 rpoN : : Tn10/F' pro lacIsqZU118 Tn5102), was constructed as described previously (Hsieh et al., 1994). The mutant plasmids were used to transform YMC109tk. All the sigma 54 mutants reported here inhibited cell growth on plates containing W-gln/IPTG, an indicator of sigma 54 protein expression (Sasse-Dwight \& Gralla, 1990). Promoter protection studies confirmed that the mutant and wild-type sigma 54 proteins were expressed at levels that allowed full promoter occupancy.

Plating test. Plasmids containing each of the mutant or wild-type sigma 54 proteins were transformed into strain 
YMC109tk and the transformed cells were screened for growth on W-arg/X-Gal plates for further comparison. Warg/X-Gal plates were prepared as described by Hsieh et al. (1994); Reitzer \& Magasanik (1986); and Smith et al. (1971) and contained $0 \cdot 01 \mathrm{mg}$ arginine $\mathrm{ml}^{-1}$ and $40 \mu \mathrm{g} \mathrm{X}-\mathrm{Gal} \mathrm{ml} \mathrm{m}^{-1}$, where arginine was the only nitrogen source. YMC109tk cells contain two copies of the sigma-54-dependent $g \ln A p 2$ promoter. One directs transcription of the glutamine synthetase gene, required for growth on minimal (W-arg) plates where arginine is the sole nitrogen source; the other is fused to the $\beta$ galatosidase gene in order to indicate $g \ln A p 2$ expression via a blue colour on X-Gal plates. The incubation conditions were as described previously (Hsieh et al., 1994).

In vivo dimethyl sulfate (DMS) footprinting, potassium permanganate probing and mRNA analysis. These three assays were all done in identical media (G-gln) containing IPTG (Hsieh et al., 1994). YMC109tk cells bearing the desired substitution plasmids were grown in G-gln media and induced with IPTG as described previously (Hsieh et al., 1994). The procedures for footprinting were as described by Hsieh \& Gralla (1994). For RNA analysis following IPTG induction, $5 \mathrm{ml}$ cells at an $\mathrm{OD}_{600}$ of $\sim 0.7-0.8$ were spun down and resuspended in $350 \mu \mathrm{l} \mathrm{SA}(0.15 \mathrm{M}$ sucrose, $0.01 \mathrm{M}$ sodium acetate), then lysed in $2 \%$ SDS/SA solution at $65^{\circ} \mathrm{C}$ for $1 \mathrm{~min}$. Sequentially, $60 \mu \mathrm{l} 2 \mathrm{M}$ sodium acetate $\mathrm{pH} 4,0.5 \mathrm{ml}$ phenol and $100 \mu \mathrm{l}$ chloroform/isoamyl alcohol $(24: 1)$ were added and the mixture shaken for $10 \mathrm{~s}$, then cooled on ice for $15 \mathrm{~min}$. Samples were treated as described previously (Hsieh et al., 1994). The $g \ln D$ primer was also used for RNA primer extension. A Bla-1 primer which hybridized to the $\beta$-lactamase gene was included as an internal control. The samples were resolved on $6 \%$ DNA sequencing gels.

Gel mobility shift assay. Ten milliliters of cells harbouring pTH7 were grown in LB medium supplemented with antibiotics and the crude cell extract was prepared as described by Tintut et al. (1994). A biotin-labelled 55 bp fragment carrying the sequence from -45 to +10 of the Rhizobium meliloti nifH promoter (Sundaresan et al., 1983) was used as a probe. Briefly, two 5'-biotin labelled 37-bp-long oligomers with 19 overlapping bases were hybridized and the ends of the duplex were filled with Klenow DNA polymerase. Protein concentrations of the crude extract were determined with the BioRad Bradford protein assay. Equal amounts of crude cell extracts containing different mutant forms of sigma 54 were incubated with binding buffer supplemented with $0.01 \mu \mathrm{g}$ poly(dI.dC) $\mathrm{ml}^{-1}, 1 \mathrm{mM}$ ATP and $3.5 \%$ (w/v) polyethylene glycol (PEG) at $30^{\circ} \mathrm{C}$ for $15 \mathrm{~min}$. Biotin-labelled probe $(3 \mathrm{pmol})$ was then added and the samples were loaded onto a $5 \%$ native polyacrylamide gel. Electrophoresis was carried out as described by Tintut et al. (1994) and the gel was then transferred to a nitrocellulose filter. The filter was then blotted by use of streptavidin/horseradish peroxidase and the signals were detected using SuperSignal Substrate (Pierce) according to the manufacturer's manual.

\section{RESULTS}

\section{Mutant construction by site-directed mutagenesis}

Single and double substitutions were introduced into the heptad repeat in Region III of sigma 54 by the M13 in vitro mutagenesis system (Taylor et al., 1985; Tintut et al., 1994). Substitution mutants in which leucines or isoleucines were changed to serines were created to remove the hydrophobic character and to maintain comparability with previous mutants created in the

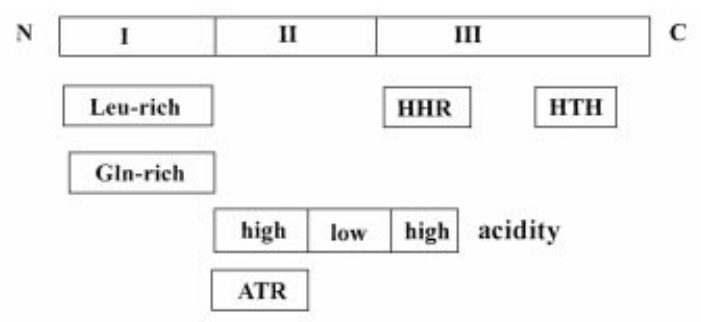

hydrophobic heptad repeat

WT



IS165

$\mathbf{S}$

IS172 S

LS179

$\mathrm{S}$

$\begin{array}{llllllll}\text { LS158/165 } & \text { S } & & \text { S } & & & \\ \text { IS165/172 } & & & \text { S } & \text { S } & & \\ \text { IS172/179 } & & & & \text { S } & & \text { S } \\ \text { LHS-3 } & \text { S } & \text { S } & \text { S } & & & \\ \text { RHS-3 } & & & & & \text { S } & \text { S } & \text { S }\end{array}$

Fig. 1. Structural domains of sigma 54 (adapted from Hsieh \& Gralla, 1994) and amino acid sequences of the substitution mutants in the sigma 54 Region III hydrophobic heptad repeat. The boxes indicate the leucine-rich region (Leu-rich), hydrophobic heptad repeat (HHR), glutamine-rich region (GInrich), acidic region (high and low acidity) and acidic trimer repeat (ATR). The sequence shows the 35 amino acids (in standard single letter code) of Region III of the sigma 54 gene. The specific substitutions introduced are indicated, either from $\mathrm{L}$ (leucine) to $\mathrm{S}$ (serine) or from I (isoleucine) to $\mathrm{S}$ (serine). The mutants are named according to the change from $L$ (or I) to $S$ and the amino acid position.

heptad repeat in Region I (Hsieh \& Gralla, 1994). Thus, we created mutants LS165, LS172, LS158/165, LS165/ 172 and LS172/179. Together with one single substitution mutant (LS179) and two triple mutants (LHS3 and RHS-3) created previously (Tintut et al., 1994), eight site-specific mutants exist with single, double or triple mutations in the heptad repeat residues of Region III (Fig. 1).

\section{Plating test on W-arg/X-Gal plates}

YMC109tk contains a $g \ln A p 2-l a c Z$ fusion, and this allows a plate assay for function of the $g \ln A p 2$ promoter via formation of a blue colour on X-Gal plates (Hsieh \& Gralla, 1994). Cells lacking a transformed functional sigma 54 protein do not grow when streaked onto agar plates containing arginine as the only nitrogen source. 
Table 1. mRNA production by mutants in the sigma 54 Region III hydrophobic heptad repeat

Radioactive mRNA transcripts obtained from the experiments described in the legend to Fig. 2 were quantified by counting. Results are expressed relative to the amount of mRNA produced by wild-type sigma 54. Values are means from two or three experiments and the maximum experimental variation was $\pm 15 \%$.

\begin{tabular}{|lc|cc|}
\hline $\begin{array}{l}\text { Sigma 54 } \\
\text { mutant }\end{array}$ & $\begin{array}{c}\text { Relative } \\
\text { amount } \\
\text { of mRNA }\end{array}$ & $\begin{array}{c}\text { Sigma 54 } \\
\text { mutant }\end{array}$ & $\begin{array}{c}\text { Relative } \\
\text { amount } \\
\text { of mRNA }\end{array}$ \\
\hline IS165 & $0 \cdot 7$ & IS165/172 & $0 \cdot 4$ \\
IS172 & $0 \cdot 8$ & IS172/179 & $0 \cdot 4$ \\
LS179 & $1 \cdot 0$ & LHS-3 & $0 \cdot 2$ \\
LS158/165 & $0 \cdot 3$ & RHS-3 & $<0 \cdot 1$ \\
\hline
\end{tabular}



Fig. 2. Determination of $g \ln A p 2$ transcripts by primer extension analysis. Lanes 1-10 contain total RNA from YMC109tk carrying wild-type sigma 54 (lane 1); carrying mutants IS165 (lane 2), IS172 (lane 3), LS179 (lane 4), LS158/165 (lane 5), IS165/172 (lane 6), IS172/179 (lane 7), LHS-3 (lane 8) and RHS-3 (lane 9); and with no sigma 54 (lane 10). A Bla-1 primer which hybridized to the $\beta$-lactamase gene was included as an internal control. The lowermost band is the hybridization probe alone.

Colony colours of cells transformed with all the single mutants in the heptad repeat of Region III were indistinguishable from that of cells transformed with wild-type sigma 54. However, the double mutants showed a light blue colony colouration, indicating lower levels of sigma-54-dependent $g \ln A p 2$ expression. Colonies of the two triple mutants showed white or no growth, which is consistent with previous reports (Tintut et al., 1994).

\section{mRNA production assayed by primer extension}

All the mutants were assayed for $g \ln A p 2$ mRNA formation in media containing glutamine (G-gln media). Growth in this medium is strongly inducing for $g \ln A p 2$
Table 2. Open complex formation by mutants in the sigma 54 Region III hydrophobic heptad repeat

Open complex levels were quantified by excising and counting the radioactive bands corresponding to the open complex formed at the $g \ln A p 2$ transcription start site. Results are expressed relative to the amount of open complex formed by wild-type sigma 54. Values are means from two or three experiments and the maximum experimental variation was $\pm 15 \%$.

\begin{tabular}{|lc|cc|}
\hline $\begin{array}{l}\text { Sigma 54 } \\
\text { mutant }\end{array}$ & $\begin{array}{r}\text { Relative } \\
\text { amount } \\
\text { of DNA } \\
\text { melting }\end{array}$ & $\begin{array}{c}\text { Sigma 54 } \\
\text { mutant }\end{array}$ & $\begin{array}{r}\text { Relative } \\
\text { amount } \\
\text { of DNA } \\
\text { melting }\end{array}$ \\
\hline IS165 & $0 \cdot 8$ & IS165/172 & $0 \cdot 8$ \\
IS172 & $0 \cdot 9$ & IS172/179 & $0 \cdot 8$ \\
LS179 & $0 \cdot 9$ & LHS-3 & $0 \cdot 2$ \\
LS158/165 & $1 \cdot 0$ & RHS-3 & $<0 \cdot 1$ \\
\hline
\end{tabular}

expression, but sigma 54 is not required for growth (Hsieh et al., 1994). This allows mRNA levels to be measured for all mutants. Primer extension experiments were performed using a $g \ln D$ probe hybridized to $g \ln A p 2$ transcript together with an internal control Bla1 probe, which hybridized to the $\beta$-lactamase gene, transcribed by sigma 70 holoenzymes (Wong et al., 1994). The levels of $g \ln A p 2$ transcript were normalized to that of $\beta$-lactamase and the results are summarized in Table 1 . The mutants with single substitutions in the heptad repeat (lanes 2, 3 and 4 in Fig. 2) produced between $70 \%$ and $100 \%$ of wild-type sigma 54 (lane 1) transcript levels. In addition, consistent with the plating test, the double mutants showed only about $30-40 \%$ of wild-type transcript levels (lanes 5-7). Our results also confirmed that the triple mutants LHS-3 and RHS-3 produced $20 \%$ or less than $10 \%$, respectively, of wildtype transcript (lanes 8 and 9 and Tintut et al., 1994). Thus, multiple hydrophobic residue substitutions lead to significant changes in the ability of the sigma 54 polymerase to produce mRNA from the $g \ln A p 2$ promoter.

\section{Open complex formation in vivo assayed by potassium permanganate footprinting}

The activating event at the $g \ln A p 2$ promoter is the NtrC/NRI-dependent conversion of a closed transcription complex to an open complex (Popham et al., 1989; Sasse-Dwight \& Gralla, 1988). The melting may be detected by preventing transcription elongation with rifampicin to trap open complexes and then using the in vivo potassium permanganate assay. In a previous study, the single substitution LS179 showed no detectable defect in DNA melting (Hsieh \& Gralla, unpublished data). All the sigma 54 mutants retained the correct position of melting, consistent with the conservation of mRNA start sites shown in Fig. 2. The degree of opening 


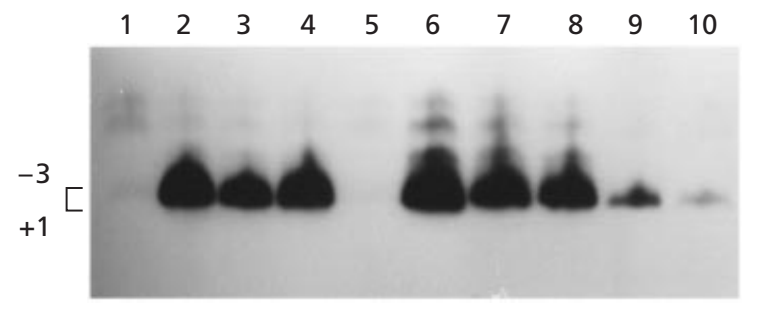

Fig. 3. Potassium permanganate probing in vivo. Lanes: 1 and 5 , no sigma $54 ; 2$, wild-type sigma $54 ; 3-4$ and 6-10, sigma 54 mutants IS165 (lane 3), IS172 (lane 4), LS158/165 (lane 6), IS165/172 (lane 7), IS172/179 (lane 8), LHS-3 (lane 9) and RHS-3 (lane 10). The -3 to +1 region of the $g \ln A p 2$ promoter is marked with a square bracket on the left.

is different for the different forms of sigma 54 polymerase. The assays were repeated two to three times and the amounts of opening were normalized to the amount of opening shown by the wild-type sigma 54 .

The collated data in Table 2 show that two triple mutants (LHS-3, RHS-3) that were most defective in mRNA production were also the most defective in open complex formation. The behaviour of single mutants IS165, IS172 and LS 179 with regard to DNA melting (lanes 3 and 4 of Fig. 3 and data not shown) was similar to that of wild-type sigma 54 (lane 2). This observation is consistent with their effect on mRNA production. However, the three double mutants LS158/165, IS165/ 172 and IS172/179 showed a tendency to retain open complex formation ability despite the lowering of mRNA levels (lanes 6-8). Because the triple mutants LHS-3 and RHS-3 do block open complex formation (lanes 9 and 10), we concluded that it is necessary to change more than two hydrophobic residues to interfere significantly with the ability to form an open complex.

\section{Analysis of DNA binding in vivo by DMS footprinting}

The altered melting and mRNA production directed by some of the mutant forms of sigma 54 could be due to altered DNA binding by the sigma 54 polymerase at the $g \ln A p 2$ promoter. We therefore performed in vivo footprinting to analyse protection of the guanine residues at $-13,-24$ and -25 from attack by DMS. It was demonstrated previously that such assays reveal promoter occupancy by assessing protection of bands near the -24 region and the -12 region of the $g \ln A p 2$ promoter (Sasse-Dwight \& Gralla, 1988, 1990).

One hundred percent promoter occupancy is considered to be the difference in signal between no sigma 54 (lane 1 in Fig. 4) and wild-type sigma 54 (lane 2). The extent of promoter occupancy is indicated by the reduced intensity of the bands near -24 and -12 compared to the -19 reference band. The significant protection of -24 compared with -19 in all hydrophobic mutant samples confirms that the mutant forms of sigma 54 in the hydrophobic region of Region III are stable enough



Fig. 4. DMS footprinting at the glnAp2 promoter. Protection patterns are shown with no sigma 54 (lanes 1 and 5); wild-type sigma 54 (lane 2); and sigma 54 mutants IS165 (lane 3), IS172 (lane 4), LS158/165 (lane 6), IS165/172 (lane 7), IS172/179 (lane 8), LHS-3 (lane 9) and RHS-3 (lane 10). The -12 and -24 regions are marked with square brackets and the -19 band with an arrow on the left.

to provide a substantial level of occupancy of the promoter. It confirms previous studies that this region does not play a significant role in -24 protection (SasseDwight \& Gralla, 1990).

These experiments also show the extent to which various hydrophobic mutants affect protection of the -12 promoter element. Since protection of the -12 element is substantial but not complete even for the wild-type sigma 54 (lane 2 in Fig. 4), the extent of protection is judged by comparison to the -19 reference band, which is not protected by sigma 54 binding to the promoter (Hsieh et al., 1994). Among the heptad repeat mutants, the most obvious defects are caused by mutants LHS-3 and RHS- 3 in lanes 9 and 10 , where the -12 to -19 ratio is much more like that in cells entirely lacking sigma 54 (lanes 1 and 5) than in cells containing wildtype sigma 54 (lane 2). However, changes in single heptad repeat residues (lanes 3 and 4) showed the same DMS footprinting patterns as the wild-type (lane 2). In addition, consistent with the mRNA production, double mutants in the heptad repeat region (lanes 6-8) showed patterns in which the -12 to -19 ratio is more similar to that from cells entirely lacking sigma 54 (lanes 1 and 5) than from cells containing wild-type sigma 54 (lane 2). The results of DMS footprinting demonstrated that progressive loss of -12 promoter recognition accompanies the progressive loss of hydrophobicity in this 




Fig. 5. Gel mobility shift assay in crude cell extracts. Lanes: 1, no sigma $54 ; 2$, wild-type sigma $54 ; 3-9$, sigma 54 mutants IS165 (lane 3), IS172 (lane 4), LS158/165 (lane 5), IS165/172 (lane 6), IS172/179 (lane 7), LHS-3 (lane 8) and RHS-3 (lane 9). H, C and $\mathrm{S}$ refer to the holoenzyme, core enzyme and sigma-54binding positions, respectively.

region. However, all the mutant forms of sigma 54 are structurally stable with regard to DNA binding, indicated by the full protection of the -24 promoter element.

\section{In vitro RNA polymerase binding assays with cell extracts}

The altered transcription site opening, mRNA production and loss of the -12 promoter recognition directed by some of the mutant forms of sigma 54 could be due to altered core-binding ability of the mutant sigma 54 polymerase. Therefore, we performed in vitro RNA polymerase binding assays to analyse different mutant forms of sigma 54. We adapted the gel mobility shift assay to use crude extracts to determine the interaction of the sigma 54 mutants with core RNA polymerase (Tintut et al., 1994). Soluble extracts from cells expressing the mutant forms of sigma 54 were incubated with the $5^{\prime}$-biotin-labelled $R$. meliloti nifH promoter probe. The resulting protein-probe complexes were then resolved on a $5 \%$ native polyacrylamide gel (Fig. 5). When sigma 54 was completely absent (host cells alone without sigma 54 expression), neither holoenzyme nor sigma 54 binding was observed (lane 1). The loss of one hydrophobic residue in Region III caused no detectable loss of core-binding ability when compared to that of wild-type sigma 54 (lanes 3 and 4 compared to lane 2). However, the loss of two hydrophobic residues in the hydrophobic heptad repeat led to a significant loss of holoenzyme formation (lanes 5-7 compared to lanes 2-4). Triple mutants LHS-3 and RHS-3 showed severe defects in the ability to produce a band corresponding to holoenzyme binding but retained the ability of free mutant sigma 54 to bind probe (lanes 8 and 9). The increased intensity of the core band in lanes 8 and 9 may be due to the relative abundance of free core polymerase, consistent with earlier reports (Tintut et al., 1994). It was noted that all the mutant proteins could bind and shift similar amounts of the promoter fragment without core RNA polymerase (Fig. 5), suggesting that the levels of expression of all the substitution mutant proteins were comparable. This finding also indicated that the decreased holoenzyme formation accompanying progressive loss of hydrophobicity is not due to protein instability.

\section{DISCUSSION}

Our aim in this work was to characterize the functional importance of the hydrophobic heptad repeat in Region III of E. coli sigma 54. Previous studies demonstrated that two triple mutants adversely affect the ability of sigma 54 to bind to core RNA polymerase (Tintut et al., 1994). However, without a systematic mutagenesis study, it was not clear whether all the hydrophobic residues are functionally important for core-binding ability. In this study, single and double mutants in leucine or isoleucine residues in the heptad repeat in Region III were created. Together with the two triple mutants created previously (Tintut et al., 1994), all the eight substitution mutants inhibited growth on Wgln/IPTG plates, an indicator of sigma 54 protein expression (Sasse-Dwight \& Gralla, 1990). Protein analysis also showed that the levels of expression of the substitution mutant proteins were similar to that of the wild-type sigma 54 (data not shown). Our experiments showed that single substitutions in the heptad repeat of Region III did not cause discernible loss of function. Double mutants retained only $30-40 \%$ of wild-type mRNA levels due to the partial loss of their ability to bind core RNA polymerase. In addition, the two triple mutants (Tintut et al., 1994) lost most, if not all, of their ability to bind core RNA polymerase, to protect the -12 promoter element of the glnAp2 promoter and to open the transcription start site. The results reported here demonstrate that the progressive destruction of hydrophobic residues in the heptad repeat of Region III leads to a progressive loss in core-RNA-polymerasebinding ability (Fig. 5), resulting in loss of -12 recognition and mRNA production.

When DNA binding, core-binding ability, -12 promoter recognition and DNA opening were assayed in the mutants, it was core-binding ability and -12 promoter recognition that showed a gradual change, whilst recognition at the -24 element remained intact. It has been reported that the domain responsible for enhancing and mediating DNA-binding activity is distinct from the core-RNA-polymerase-binding domain (Cannon et al., 1997). Our results are consistent with this model and provide evidence that the mutant forms of sigma 54 altered in the core-binding domain do not influence DNA binding in the -24 promoter region. Our results further supported the earlier suggestion that sigma 54 is responsible for most of the close contacts detected between sigma 54 holoenzyme and promoter DNA (Buck \& Cannon, 1992; Cannon et al., 1995a). However, the formation of an activatable promoter complex is dependent upon a critical interaction between sigma 54 and core RNA polymerase and the 
proper positioning of holoenzyme on the promoter elements.

It is noteworthy that the progressive loss of function and -12 promoter element recognition is not always accompanied by a loss of ability to form open complexes in vivo. The double mutants in the heptad repeat of Region III still retained nearly normal ability to form open complexes in vivo, but showed severe loss of ability to bind core RNA polymerase, substantially eliminating -12 promoter recognition. The double mutants retained mRNA production to only $30-40 \%$ of the wild-type level. It has been demonstrated that sigma 54 alone is unable to function in the process of open complex formation unless bound as holoenzyme (Buck \& Cannon, 1992). We also showed here that double mutations in Region III led to a partial loss of holoenzyme formation. Therefore, it seems that even though the double mutants only retained partial core-binding ability, the holoenzyme they form is capable of DNA melting. However, these open complexes do not seem to be fully functional. It is likely that the defect in these double mutants alters the structure required for activator-dependent DNA melting. The loss of function and -12 promoter element recognition not always being accompanied by a loss of ability to form open complexes in vivo was also observed in double mutants in Region I (Hsieh \& Gralla, 1994). The double mutant LS26/33 or multiple leucine mutant was found to convert the sigma 54 polymerase to an enhancer-independent enzyme (Syed \& Gralla, 1997, 1998; Wang et al., 1995). Therefore, we cannot exclude the possibility that the double mutants in the heptad repeat of Region III also contain the enhancer-bypass property, which may unlock the melting potential of the polymerase. It will be of great interest to test whether these double mutants behave as enhancer-independent enzymes. The results will also provide insight into how sigma 54 holoenzyme responds to the activator $\mathrm{NtrC}$ in the enhancer-like transcription initiation.

Previous work has suggested that only a small region of Region III of sigma 54 plays an essential role in binding the core polymerase (Cannon et al., 1995b; Tintut et al., 1994; Wong et al., 1994). This region contains three motifs of interest: hydrophobic, acidic and a short stretch between residues 175 and 180 (Tintut et al., 1994; Tintut \& Gralla, 1995). Our present results further demonstrate that it takes more than one hydrophobic residue in the heptad repeat motif of Region III to affect ability to bind core RNA polymerase. In conclusion, we confirmed here by site-directed mutagenesis that the structural features of the hydrophobic heptad repeat of Region III are essential for core-RNA-polymerasebinding ability in the enhancer-dependent transcription pathway.

\section{ACKNOWLEDGEMENTS}

We thank Dr Jay D. Gralla for comments and gifts of sigma 54 mutants LHS-3, RHS-3 and LS179. This work was supported by a grant from the National Science Council of the Republic of China, NSC86-2314-B-040-014, and from the Chung Shan Medical and Dental College Research Fund, CSMC 85-OMA-036.

\section{REFERENCES}

Austin, S. \& Dixon, R. A. (1992). The prokaryotic enhancer binding protein NTRC has ATPase activity which is phosphorylation and DNA dependent. EMBO J 11, 2219-2228.

Austin, S., Buck, M., Cannon, W., Eydmann, T. \& Dixon, R. (1994). Purification and in vitro activities of the native nitrogen fixation control proteins NifA and NifL. J Bacteriol 176, 3460-3465.

Birkmann, A. \& Bock, A. (1989). Characterization of a cisregulatory element necessary for formamate induction of the formate dehydrogenase gene $(f d h F)$ of Escherichia coli. Mol Microbiol 3, 187-195.

Buck, M. \& Cannon, W. (1992). Specific binding of the transcription factor sigma-54 to promoter DNA. Nature 358, 422-424.

Cannon, W., Claverie-Martin, F., Austin, S. \& Buck, M. (1993). Core RNA polymerase assists binding of the transcription factor $\sigma^{54}$ to promoter DNA. Mol Microbiol 8, 287-298.

Cannon, W., Austin, S., Moore, M. \& Buck, M. (1995a). Identification of close contacts between the $\sigma^{\mathrm{N}}\left(\sigma^{54}\right)$ protein and promoter DNA in closed promoter complexes. Nucleic Acids Res 23, 351-356.

Cannon, W., Missailids, S., Smith, C., Cottier, A., Austin, S., Moore, M. \& Buck, M. (1995b). Core RNA polymerase and promoter DNA interactions of purified domains of $\sigma^{\mathrm{N}}$ : bipartite functions. J Mol Biol 248, 781-803.

Cannon, W. V., Chaney, M. K., Wang, X.-Y. \& Buck, M. (1997). Two domains within $\sigma^{\mathrm{N}}\left(\sigma^{54}\right)$ cooperate for DNA binding. Proc Natl Acad Sci USA 94, 5006-5011.

Gross, C. A., Lonetto, M. \& Losick, R. (1992). Bacterial sigma factors. In Transcriptional Regulation, pp. 129-195. Edited by S. L. McKnight \& K. R. Yamamoto. Cold Spring Harbor, NY: Cold Spring Harbor Laboratory.

Guo, Y. \& Gralla, J. D. (1997). DNA-binding determinants of sigma 54 as deduced from libraries of mutations. J Bacteriol 179, 1239-1245.

Hsieh, M. \& Gralla, J. D. (1994). Analysis of the N-terminal leucine heptad and hexad repeats of sigma 54. J Mol Biol 239, 15-24.

Hsieh, M., Tintut, Y. \& Gralla, J. D. (1994). Functional roles for the glutamines within the glutamine-rich region of the transcription factor sigma 54. J Biol Chem 269, 373-378.

Merrick, M. \& Chambers, S. (1992). The helix-turn-helix motif of $\sigma^{54}$ is involved in recognition of the -13 promoter region. $J$ Bacteriol 174, 7221-7226.

Merrick, M. J., Gibbons, J. \& Toukdarian, A. (1987). The nucleotide sequence of the sigma factor gene ntrA $(r p o N)$ of Azotobacter vinelandii: analysis of conserved sequences in NtrA proteins. Mol Gen Genet 210, 323-330.

Morris, L., Cannon, W., Claverie-Martin, F., Austin, S. \& Buck, M. (1994). DNA distortion and nucleation of local DNA unwinding within sigma 54 holoenzyme closed promoter complexes. J Biol Chem 269, 11563-11571.

Popham, D. L., Szeto, D., Keener, J. \& Kustu, S. (1989). Function of a bacterial activator protein that binds to transcriptional enhancers. Science 243, 629-635.

Reitzer, L. J. \& Magasanik, B. (1986). Transcription of $g \ln A$ in $E$. coli is stimulated by activator bound to sites far from the promoter. Cell 45, 785-792.

Sasse-Dwight, S. \& Gralla, J. D. (1988). Probing the Escherichia 
coli glnALG upstream activation mechanism in vivo. Proc Natl Acad Sci USA 85, 8934-8938.

Sasse-Dwight, S. \& Gralla, J. D. (1990). Role of eukaryotic-type functional domains found in the prokaryotic enhancer receptor factor sigma 54. Cell 62, 945-954.

Smith, G. R., Halpern, Y. S. \& Magasanik, B. (1971). Genetics and metabolic control of enzymes responsible for histidine degradation in Salmonella typhimurium. 4-imidazolone-5-propionate amidohydrolase and $\mathrm{N}$-formimino-L-glutamate formiminohydrolase. J Biol Chem 246, 3320-3329.

Su, W., Porter, S., Kustu, S. \& Echols, H. (1990). DNA-looping and enhancer activity: association between DNA-bound NtrC activator and RNA polymerase at the bacterial $g \ln A$ promoter. Proc Natl Acad Sci USA 87, 5504-5508.

Sundaresan, V., Jones, J. D. G., Ow, D. W. \& Ausubel, F. M. (1983). Klebsiella pneumoniae nifA product activates the Rhizobium meliloti nitrogenase promoter. Nature 301, 728-732.

Syed, A. \& Gralla, J. D. (1997). Isolation and properties of enhancer-bypass mutants of sigma 54. Mol Microbiol 23, 987-995.

Syed, A. \& Gralla, J. D. (1998). Identification of an N-terminal region of sigma 54 required for enhancer responsiveness. $J$ Bacteriol 180, 5619-5625.

Taylor, J. W., Ott, J. \& Eckstein, F. (1985). The rapid generation of oligonucleotide-directed mutation at high frequency using phosphothioate-modified DNA. Nucleic Acids Res 13, 8764-8785.

Taylor, M., Butter, R., Chambers, S., Casimiro, M., Badii, F. \&
Merrick, M. (1996). The RpoN-box of the RNA polymerase sigma factor $\sigma^{54}$ plays a role in promoter recognition. Mol Microbiol 22, $1045-1054$.

Tintut, Y. \& Gralla, J. D. (1995). PCR mutagenesis identifies a polymerase-binding sequence of sigma 54 that includes a sigma 70 homology region. J Bacteriol 177, 5818-5825.

Tintut, Y., Wong, C. W., Jiang, Y., Hsish, M. \& Gralla, J. D. (1994). Polymerase binding using a strongly acidic hydrophobic-repeat region of sigma 54. Proc Natl Acad Sci USA 91, 2120-2124.

Wang, J., Syed, A., Hsieh, M. \& Gralla, J. D. (1995). Converting Escherichia coli RNA polymerase into an enhancer-responsive enzyme - role of a $\mathrm{NH}_{2}$-terminal leucine patch in sigma 54 . Science 270, 992-994.

Weiss, D. S., Klose, K. E., Hoover, T. R., North, A. K., Porter, S. C., Wedel, A. B. \& Kustu, S. (1992). Prokaryotic enhancers. In Transcriptional Regulation, pp. 667-694. Edited by S. L. McKnight \& K. R. Yamamoto. Cold Spring Harbor, NY : Cold Spring Harbor Laboratory.

Wong, C. W., Tintut, Y. \& Gralla, J. D. (1994). The domain structure of sigma 54 as determined by analysis of a set of deletion mutants. J Mol Biol 236, 81-90.

Wyman, C., Rombel, I., North, A. K., Bustamante, C. \& Kustu, S. (1997). Unusual oligomerization required for activity of NtrC, a bacterial enhancer-binding protein. Science 275, 1658-1661.

Received 22 April 1999; revised 28 July 1999; accepted 6 August 1999. 